\title{
Gd-EOB-DTPA dynamic contrast-enhanced magnetic resonance imaging is more effective than enhanced 64-slice CT for the detection of small lesions in patients with hepatocellular carcinoma
}

\author{
Jiangfa Li, MM ${ }^{a, b}$, Xiaoqing Li, MM ${ }^{a, c}$, Jun Weng, MM ${ }^{a, b}$, Liping Lei, BD ${ }^{a, d}$, Jianhua Gong, MM ${ }^{a, e}$, \\ Junyi Wang, MM ${ }^{a, f}$, Zhenghang Li, MM ${ }^{a, b}$, Longmiao Zhang, BD ${ }^{a, b}$, Songqing He, MD ${ }^{a * *}$
}

\begin{abstract}
This study aimed to compare the sensitivity and accuracy for the detection of small lesions in patients with hepatocellular carcinoma (HCC) using gadolinium-ethoxybenzyl-diethylenetriamine pentaacetic acid (Gd-EOB-DTPA) dynamic contrast-enhanced magnetic resonance imaging (DCE-MRI) and 64-slice computed tomography (CT) enhanced scanning, and to evaluate the necessity to perform MRI in patients diagnosed with HCC by CT.

The clinical data from 209 patients with HCC diagnosed prior to surgery in the Affiliated Hospital of Guilin Medical University, China were retrospectively analyzed. The 64-slice dynamic contrast-enhanced multi-detector CT (MDCT) and 3.0T Gd-EOB-DTPA DCE $\mathrm{MRI}$ procedures were successively carried out on all patients who were enrolled in a self-controlled study including detection and diagnosis of HCC lesions by MRI and CT, respectively.

A total of 243 lesions were detected and both imaging methods could accurately detect lesions of diameter $>2 \mathrm{~cm}$. For lesions $<2$ $\mathrm{cm}, \mathrm{MRI}$ detected 47, while CT detected 25 lesions indicating that the detection rate of MRI was 88\% higher than that of CT. In addition, MRI detected lesions in 15 cases (7.81\% in the total of 209 cases) that were not diagnosed by CT. Among these cases, 2 patients were diagnosed to have no lesion by CT.

Gd-EOB-DTPA DCE-MRI performed as a routine check prior to surgery in HCC patients can improve the detection of small HCC lesions.

Abbreviations: 3DVIBE $=$ three-dimensional volume interpolated breath-hold examination, $\mathrm{CT}=$ computed tomography, $\mathrm{DCE}=$ dynamic contrast-enhanced, DN = dysplastic nodules, Gd-EOB-DTPA = gadolinium-ethoxybenzyl-diethylenetriamine pentaacetic acid, HCC = hepatocellular carcinoma, HGDN = high-grade dysplastic nodules, MDCT = multi-detector CT, MRI = magnetic resonance imaging.
\end{abstract}

Keywords: dynamic contrast-enhanced magnetic resonance imaging, Gd-EOB-DTPA, hepatocellular carcinoma, small intrahepatic lesion

\footnotetext{
Editor: Simona Gurzu.

Jiangfa Li, Xiaoqing Li, and Jun Weng contributed equally to this work.

Financial support: This work was supported in part by the National Natural Science Foundation of China (No. 31370917, No.81430014 and No. 81160066), the project to improve middle-aged and young teacher's basic abilities in the Universities of Guangxi (KY2016YB326), and the Guangxi Key Laboratory of Molecular Medicine in Liver Injury and Repair (NO.16-140-46-04), 111 Project (D17011) and Guangxi BaGui Scholars.

The authors have no conflicts of interest to disclose.

${ }^{a}$ Department of Hepatobiliary and Pancreatic Surgery, The First Affiliated Hospital of Guangxi Medical University, Nanning 530021, China, ${ }^{b}$ Department of Hepatobiliary and Pancreatic Surgery, Affiliated Hospital of Guilin Medical University, Guilin 541001, China, ' Department of General Surgery, The Third Affiliated Hospital of Henan University of Science and Technology, Luoyang 471003, China, ${ }^{d}$ Department of Infectious Diseases, The Second Affiliated Hospital of Guilin Medical University, Guilin 541001, China, ${ }^{e}$ Department of Hepatobiliary Surgery, The First College of Clinical Medical Science, China Three Gorges University, Yichang, Hubei 443003, China, ${ }^{f}$ Department of Gastroenterology, Hospital of Xiangshui County, Xiangshui 224600, China.

* Correspondence: Songqing He, Department of Hepatobiliary and Pancreatic Surgery, The First Affiliated Hospital of Guangxi Medical University, Nanning 530021, China (e-mail: dr_hesongqing@163.com).
}

Copyright (c) 2018 the Author(s). Published by Wolters Kluwer Health, Inc.

This is an open access article distributed under the terms of the Creative Commons Attribution-Non Commercial License 4.0 (CCBY-NC), where it is permissible to download, share, remix, transform, and buildup the work provided it is properly cited. The work cannot be used commercially without permission from the journal. Medicine (2018) 97:52(e13964)

Received: 6 February 2018 / Received in final form: 20 November 2018 / Accepted: 10 December 2018

http://dx.doi.org/10.1097/MD.0000000000013964 


\section{Introduction}

Hepatocellular carcinoma (HCC) is a highly malignant cancer, which is often discovered in advanced stages in many patients. With the exception of liver transplantation, surgery is the preferred treatment option. After radical surgery, HCC frequently recurs for which prognosis is poor in most patients. Intrahepatic metastasis can also occur in some patients prior to surgery but these lesions are difficult to be detected and often not found. The detection of intra-hepatic metastases prior to surgery has significant correlation with the determination of Barcelona staging in patients with HCC, the formulation of surgical plans, and patient prognosis.

Gadolinium-ethoxybenzyl-diethylenetriamine pentaacetic acid (Gd-EOB-DTPA) dynamic contrast-enhanced magnetic resonance imaging (DCE-MRI) is an effective method for the detection of small intra-hepatic lesions that cannot be diagnosed using computed tomography (CT). This can change the presurgical Barcelona staging of some patients. Therefore, the diagnosis of small intra-hepatic lesions as early as possible prior to surgery and accurate detection of small lesions to be excised during surgery can reduce tumor recurrence and increase overall survival. ${ }^{[1]}$ As the preferred radiological detection method for the diagnosis of HCC, enhanced CT often fails to detect small lesions in the liver. DCE-MRI is a superior technique to enhanced CT for the detection of small intra-hepatic lesions. ${ }^{[1-6]}$ Huppertz et al ${ }^{[2]}$ demonstrated that liver specific contrast agents increase the hepatic parenchymal enhancement signals during the hepatobiliary specific phase, thus improving the display of focal liver lesions, particularly small lesions with diameters $<1 \mathrm{~cm}$. In qualitative diagnosis, $\mathrm{Kudo}^{[3]}$ showed that the low signals in the delayed phase are comparative features and an independent sign of malignant liver cirrhosis, while equal signals and high signals indicate benign lesions.

DCE-MRI shows higher sensitivity, specificity, and accuracy compared to enhanced multi-detector CT (MDCT) for the detection and diagnosis of liver lesions and can also detect lesions at an earlier stage. ${ }^{[7,8]} \mathrm{A}$ large number of studies have confirmed the sensitivity, specificity, and accuracy of DCE-MRI in the diagnosis of HCC and metastatic tumors as superior to MDCT. ${ }^{[9-11]}$ Golfieri et $\mathrm{al}^{[4]}$ found that DCE-MRI additional scanning hepatocyte phases exhibited a higher sensitivity in diagnosis of atypical high-grade dysplastic nodules (HGDN) and early-stage HCC compared to commonly used enhanced MRI. Gd-EOB-DTPA is a new liver specific contrast agent that not only shows characteristics of extra-cellular contrast agents (dynamic stage) but also demonstrates those of liver specific contrast agents (static stage). Gd-EOB-DTPA is excreted from the body through the kidney or biliary system which benefits detection and qualitative diagnosis of lesions. ${ }^{[12]}$ Gd-EOB-DTPA enhanced MRI is superior to MDCT and MRI enhanced using other contrast agents for the detection and qualitative diagnosis of liver lesions. ${ }^{[13-15]}$

For liver lesions that are difficult to diagnose by CT, further detection is advised using enhanced MRI. ${ }^{[16,17]}$ However, whether it is necessary to detect patients who have been definitely diagnosed with HCC by enhanced CT to further be detected using enhanced MRI has not yet been investigated. This study aimed to compare the sensitivity and accuracy of Gd-EOB-DTPA DCE-MRI and enhanced 64-slice CT for the diagnosis of intrahepatic lesions in HCC patients, and to evaluate the necessity to perform MRI in patients diagnosed with HCC by MDCT.

\section{Materials and methods}

\subsection{Patients}

In the Affiliated Hospital of Guilin Medical College (Guilin, China), 209 patients with HCC diagnosed using established methods ${ }^{[18]}$ prior to surgery from April 2012 to April 2016 were recruited to the study. Of these patients, 153 were male and 65 were female. All patients were between 28 and 72 years old with an average age of $53.52 \pm 12.88$ years and a median age of 55 years. The diameter of lesions ranged from 0.5 to $20.5 \mathrm{~cm}$. Twenty-six patients' cases were diagnosed with intra-hepatic metastasis and 34 of these lesions were confirmed by post-operative pathology. The research was approved by the Ethics Committee of the Affiliated Hospital and all patients had given informed consent to participate in the study. CT and MRI examinations were performed successively for each patient before surgery. All patients were enrolled in a self-controlled study including detection and diagnosis of HCC lesions by MRI and CT.

\subsection{Inclusion and exclusion criteria}

2.2.1. Inclusion criteria. Those patients who were (1) diagnosed with HCC prior to surgery, (2) subjected to MDCT and Gd-EOBDTPA DCE-MRI detection, and (3) underwent surgical treatment and had pathological outcomes, were included in the study.

2.2.2. Exclusion criteria. Those patients who were (1) diagnosed with metastatic HCC, (2) not subjected to enhanced CT or enhanced MRI, and (3) did not have pathological data, were excluded in the study.

\subsection{Detection instruments}

The 64-slice spiral CT manufactured by GE (United States) was used in MDCT, and the 3.0 T MRI system manufactured by Siemens, Germany was utilized in MRI.

\subsection{Detection reagents}

In MDCT and MRI, $300 \mathrm{mgL} / \mathrm{mL}$ iopromide and Gd-EOB-DTPA $(10 \mathrm{~mL} / \mathrm{bottle})$ produced by Bayer Medical Health Co., Ltd. (Germany) were used. The Gd-EOB-DTPA was a new liverspecific contrast agent and the concentration was $0.25 \mathrm{~mol} / \mathrm{L}$.

\subsection{Detection methods}

For MDCT, a light speed VCT 64-slice spiral CT manufactured by GE (United States) was utilized in the plain scan and enhanced scan modes on all patients. Patients were fasted for $6 \mathrm{~h}$ before detection and drank $1000-1500 \mathrm{~mL}$ of clear water $30 \mathrm{~min}$ before the scan to fill the gastro-intestinal tract. The plain scan was followed by the enhanced scan. Scanning was conducted at a voltage of $120 \mathrm{kV}$ using the auto-milliampere technique with a noise index of 8. Both the slice thickness and slice gap were $5 \mathrm{~mm}$. In routine secondary thinning and reconstruction, the slice thickness and slice gap for reorganization were $1.25 \mathrm{~mm}$ to allow multi-planar reconstruction.

The contrast agent used was $95 \mathrm{~mL}$ of $300 \mathrm{mgI} / \mathrm{mL}$ iopromide produced by Bayer Medical Health Co., Ltd. (Germany) and injected at a rate of 3-4 mL/s using a high-pressure injector manufactured by E-Z-EM (United States). By employing smart technology, the abdominal aorta was detected. When the threshold reached $150 \mathrm{HU}$, the hepatic arterial phase (the advanced arterial phase) was scanned and the scanning of 
venous phase was delayed for $30 \mathrm{~s}$. The scanning lasted for 3-5 s on each occasion. During the scan, patients were required to hold their breath. If necessary, the whole liver was scanned for an additional $3-5 \mathrm{~min}$.

DCE-MRI magnetic resonance was carried out using a Verio 3.0T MRI system manufactured by Siemens (Germany) with a 12channel phased array coil. The contrast agent was $0.25 \mathrm{~mol} / \mathrm{L} \mathrm{Gd}-$ EOB-DTPA $(10 \mathrm{~mL} /$ bottle) produced by Bayer Medical Health Co., Ltd. (Germany). Before detection, patients were trained to breath uniformly and hold breath reasonably to coordinate scan and to ensure image quality and uniformity of the scanning slices. The respiratory-gating technique was used during detection. The patients were scanned from diaphragm to inferior pole of kidneys, including the whole liver. Regular plain scanning and Gd-EOBDTPA enhanced scanning including T2WI, T1-VIBE in- and outof-phase scanning, DWI scanning, and T1-VIBE fat suppression scan in turn were carried out on all patients. Afterwards, three phases (arterial, portal venous, and equilibrium phases) of GdEOB-DTPA DCE scan and delayed parenchymal phase scan were conducted based on T1-VIBE fat suppression scan.

In the Gd-EOB-DTPA DCE scan, after peripheral venous injection of $0.025 \mathrm{mmol} / \mathrm{kg}$ Gd-EOB-DTPA at the rate of $3 \mathrm{~mL} / \mathrm{s}, 20 \mathrm{~mL}$ of normal saline was injected at the same rate to wash the catheter. Three-dimensional volume interpolated breath-hold examination (3D VIBE) was conducted at periods between 18 and 23s (arterial phase), 45-50s (portal venous phase), and $120 \mathrm{~s}$ (equilibrium phase) after injecting Gd-EOB-DTPA. Axial delayed scanning was carried out by delaying $5,10,20$, and $30 \mathrm{~min}$ and then the oblique coronal scan was delayed for 20 and $30 \mathrm{~min}$, as shown in Table 1.

\subsection{Image analysis}

The obtained images were independently assessed by 2 radiologists holding titles higher than vice director in a double-blind manner. For typical lesions of HCC, the multislice spiral CT or Gd-EOB-DTPA enhanced MRI showed that the tumor lesions with a diameter $>1 \mathrm{~cm}$ were inhomogeneously enhanced in the arterial phase and rapidly washed out in the venous or delayed phases. ${ }^{[18]}$

\subsection{Statistical analysis}

The data in the two groups were compared using the Chi-square test with a threshold of $P<.05$ for statistical significance. The SPSS19.0 software was used for statistical analysis.

\section{Results}

In the 209 patients preliminarily diagnosed with HCC prior to surgery, 243 lesions were found using MRI indicating that more

\section{Table 1}

MRI scanning sequences and parameters.

\begin{tabular}{lccc}
\hline Scanning parameters & TSE T2WI & 3D VIBE & 2D GRE T1WI \\
\hline TR $(\mathrm{ms})$ & 2500.00 & 4.87 & 170.00 \\
TE $(\mathrm{ms})$ & 104.00 & $2.35-2.48$ & 5.00 \\
Matrix size & $277 \times 384$ & $167 \times 256$ & $180 \times 256$ \\
Flip angle & 150 & 10 & 70 \\
FOV & $36 \times 36$ & $27 \times 27$ to $36 \times 36$ & $36 \times 36$ \\
Slice thickness & 5.0 & $2.5 \sim 5.0$ & 5.0 \\
Slice gap & 1.0 & - & 1.0 \\
\hline
\end{tabular}

3D VIBE $=$ three-dimensional volume interpolated breath-hold examination, GRE T1W = gradient-echo T1-weighted imaging, FOV = field of view, TE = time of echo, TR=time of repetition, TSE T2W1 = turbo spin echo T2-weighted imaging.
Table 2

Diagnosis of intrahepatic lesions with different sizes.

\begin{tabular}{lcccc}
\hline & \multicolumn{4}{c}{ The number of detected lesions } \\
\cline { 2 - 5 } Item & $\mathbf{5} \mathbf{c m}$ & $\mathbf{2 - 5} \mathbf{c m}$ & $<\mathbf{2} \mathbf{c m}$ & Total \\
\hline CT & 123 & 73 & $25^{\#}$ & $221^{\# \#}$ \\
MRl & 123 & 73 & 47 & 243 \\
\hline
\end{tabular}

$\mathrm{CT}=$ computed tomography, $\mathrm{MRI}=$ magnetic resonance imaging

$\# P<.001$.

$\#$ \# $P<.001$.

lesions $(9.95 \%, 22 / 221)$ were found compared with 221 lesions detected using CT $(P<.001)$. The data are summarized in Table 2 and Figure 1. Compared to CT, MRI could detect additional lesions $<2 \mathrm{~cm}$ of diameter in 15 patients $(7.18 \%$ of 209) $(P<.001)$. Amongst these 15 patients, 2 patients (AFP $>100 \mathrm{ng} /$ $\mathrm{mL}$ ) were diagnosed without lesion using $\mathrm{CT}$, while lesions were found by MRI (diameter was $<2 \mathrm{~cm}$ ) and verified as HCC according to post-operative pathological findings as summarized in Table 3 and Figure 1.

Tumors with diameters $>2 \mathrm{~cm}$ can be detected using MRI or CT. For the detection of tumors $<2 \mathrm{~cm}, 47$ lesions were found using MRI, while only 25 lesions were detected by CT indicating that more lesions (22, accounting for $88 \%$ of 25$)$ were detected by MRI than CT, as shown in Table $2(P<.001)$. Table 4 shows the diagnosis rate of MRI. Diagnostic accordance rates of patients with HCC and detected lesions using Gd-EOB-DTPA DCE-MRI were 97.61\% (204/209) and 96.29\% (234/243), respectively.

\section{Discussion}

The study indicated that the number of detected lesions differed significantly using the two investigative methods $(P<.05)$. Kim et $\mathrm{al}^{[1]}$ found that Gd-EOB-DTPA DCE-MRI detected 74 more HCC lesions in 53 patients $(16.4 \%$ of the total 323$)$ compared to MDCT. Maiwald et al ${ }^{[19]}$ showed that about 20 more suspicious HCC lesions were detected using Gd-EOB-DTPA DCE-MRI compared with MDCT. Gd-EOB-DTPA DCE-MRI shows higher sensitivity, specificity, and accuracy in the detection and diagnosis of HCC lesions than MDCT, and can find lesions at an early stage allowing for qualitative diagnosis. ${ }^{[7,8]}$

Some studies have demonstrated that Gd-EOB-DTPA DCEMRI significantly improves the sensitivity and diagnostic accuracy for HCC compared with single multi-slice spiral CT. ${ }^{[20]}$ A larger number of studies have further verified that DCE-MRI has obvious advantages in sensitivity, specificity, and accuracy for the diagnosis of HCC and metastatic tumors compared with MDCT. ${ }^{[9-11]}$ Gd-EOB-DTPA DCE-MRI can discover small lesions in liver that cannot be found using MDCT in patients with HCC which is beneficial for formulating surgical plans and reducing post-operative recurrence. ${ }^{[1]}$ Gd-EOB-DTPA ECE-MRI therefore has significant clinical value in early-stage detection, diagnosis, and treatment of lesions in HCC patients.

This study demonstrates that the number of liver lesions $<2 \mathrm{~cm}$ in diameter detected by Gd-EOB-DTPA DCE-MRI is significantly more than those detected by MDCT (47 and 25 lesions were found by using the former and the latter, respectively, $P<.001)$. Post-operative pathology of 2 patients (four lesions) showed atypical dysplastic nodules (DN). At present, hepatoma nodules $(<2 \mathrm{~cm})$ with low blood supply is defined as small HCC according to the international medical community. Some studies have 

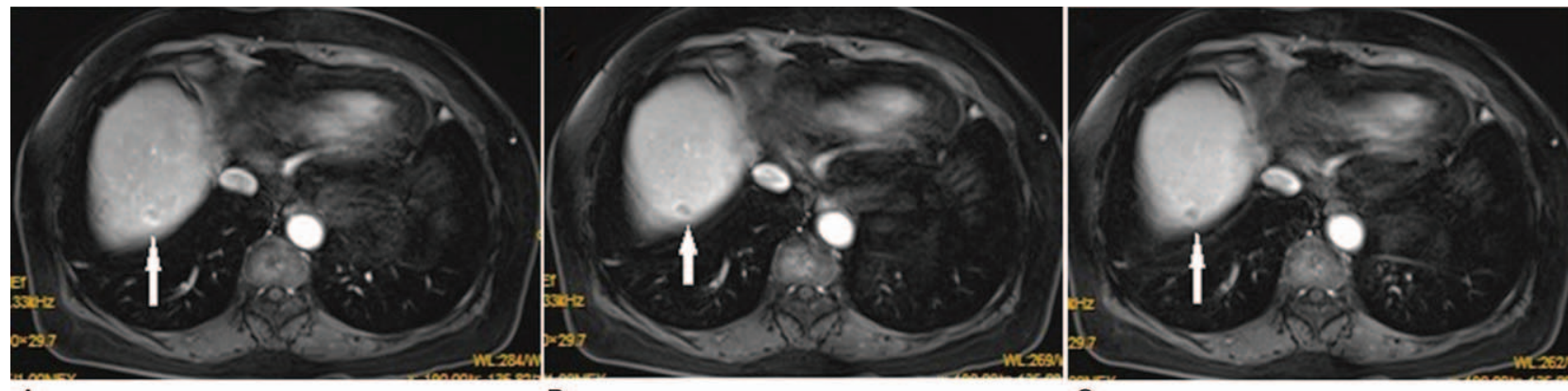

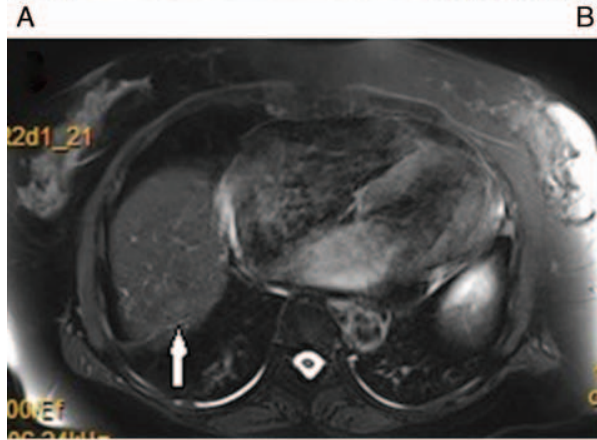

D
B

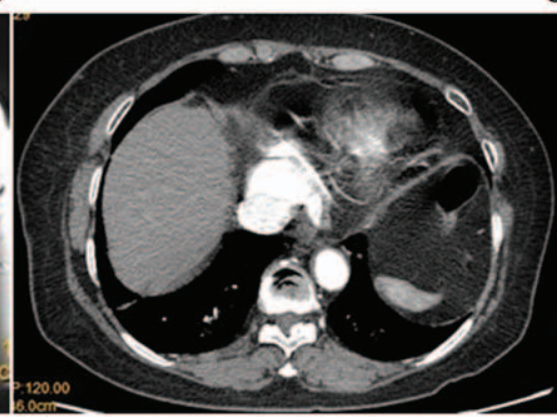

$\mathrm{C}$

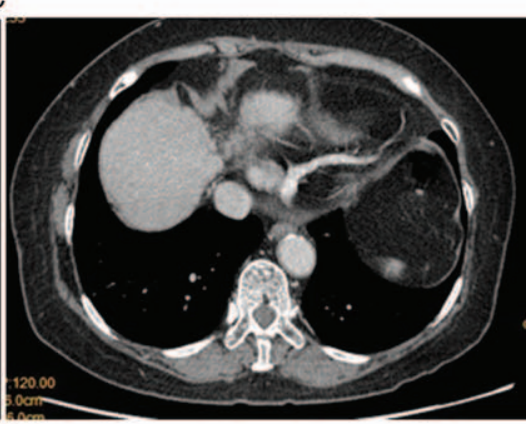

$\mathrm{E}$

$\mathrm{F}$

Figure 1. A lesion was found by MRI in the upper and posterior segments of the right hepatic lobe: A, obvious enhancement appearances during the arterial phase; $\mathrm{B}$, heterogeneous enhancement during the venous phase; $\mathrm{C}$, apparent fading of early enhancement in the delayed phase; $\mathrm{D}$, low signal change at the hepatocyte specific phase. No lesion was detected using CT: E, no abnormal density shadow appearing in the enhanced scan during the arterial phase; F, no apparent abnormal density shadow found in the venous phase.

shown that Gd-EOB-DTPA DCE-MRI is superior to MDCT in diagnosis and detection of small HCC. ${ }^{[6,15,21]}$ Inoue et al ${ }^{[15]}$ also found that the liver-specific strengthening characteristics in the hepatocyte phase of DCE-MRI has certain significance for diagnosing HCC lesions $(<2 \mathrm{~cm})$ and atypical $\mathrm{DN}$, while the identification of the lesions needs to be further studied.

Several existing reports have verified that Gd-EOB-DTPA DCE-MRI can improve the detection ability for HCC less for early stage lesion with a diameter $<2 \mathrm{~cm}$. In excised liver specimens from 30 patients with HCC, 23 out of 24 cases who had hepatoma nodules with low or slightly low signals in liver images using MRI were diagnosed as early-phase HCC, while only one case was wrongly diagnosed as atypical DN or regenerative nodule (RN). Moreover, in the 6 cases with nodules showing high signals, 5 cases were diagnosed correctly and only one case was incorrectly diagnosed as early-stage HCC, showing a $93 \%$ (28/30) diagnostic accordance rate. ${ }^{[22]}$ In addition, Golfieri et $\mathrm{al}^{[4]}$ found that DCE-MRI additionally scanning the hepatocyte phase shows a higher sensitivity for the diagnosis of HGDN and early-stage HCC than commonly enhanced MRI.

\section{Table 3}

Distribution of cases with different numbers of lesions in livers.

\begin{tabular}{lccccc}
\hline & \multicolumn{5}{c}{ Number of cases with different lesions } \\
\cline { 2 - 6 } Item & $\begin{array}{c}\text { No lesions } \\
\text { found }\end{array}$ & $\begin{array}{c}\text { Single } \\
\text { lesion }\end{array}$ & $\begin{array}{c}\text { Double } \\
\text { lesions }\end{array}$ & $\begin{array}{c}\text { Three } \\
\text { lesions }\end{array}$ & $\begin{array}{c}\text { Four and } \\
\text { above lesions }\end{array}$ \\
\hline CT & 2 & $194^{\#}$ & $12^{\# \#}$ & 1 & 0 \\
MRI & 0 & 183 & 20 & 4 & 2 \\
\hline
\end{tabular}

$\mathrm{CT}=$ computed tomography, $\mathrm{MRl}=$ magnetic resonance imaging.

${ }^{\#} P<.001$.

\#\# $P<.001$
In this study, Gd-EOB-DTPA DCE-MRI was found to be able to diagnose HCC patients who were missed using enhanced 61slice CT. Two patients whose AFP was $>100 \mathrm{ng} / \mathrm{mL}$ were diagnosed without lesions, and HCC lesions were detected using Gd-EOB-DTPA DCE-MRI. The rate of missed diagnosis using CT was $0.96 \%(2 / 209)$. Maiwald et $\mathrm{al}^{[19]}$ found that among 50 suspicious patients with HCC, there were no obvious liver lesions found in a patient through multi-slice spiral enhanced CT, while an HCC lesion was diagnosed by Gd-EOB-DTPA DCE-MRI. Although MDCT is still the preferred method for detection of focal liver lesions, when lesions in four-phased MDCT or DCEMRI images cannot be diagnosed, it is suggested to use these two methods simultaneously as stated in the consensus report of the fourth International Forum for Gd-EOB-DTPA. MDCT is the first choice which should then be combined with Gd-EOB-DTPA DCE-MRI when lesions are difficult to diagnose. ${ }^{[16]}$ However, this study showed that focal liver lesions, especially HCC nodules whose diameter is $<2 \mathrm{~cm}$, are likely to be missed in the diagnosis by using MDCT. This is the advantage of using the liver-specific

\section{Table 4}

\section{Post-operative pathology.}

\begin{tabular}{lcccc}
\hline & \multicolumn{4}{c}{ Case diagnosis } \\
\cline { 2 - 5 } Item & HCC & $\begin{array}{c}\text { Inflammatory } \\
\text { pseudotumor }\end{array}$ & FNH & $\begin{array}{c}\text { Untypical } \\
\text { DN }\end{array}$ \\
\hline Patients & 204 & 2 & 3 & $\begin{array}{c}\text { 2 (the main lesion is } \\
\text { diagnosed with HCC) } \\
\text { Lesions }\end{array}$ \\
\hline
\end{tabular}

$\mathrm{DN}=$ dysplastic nodules, $\mathrm{FNH}=$ focal nodular hyperplasia, $\mathrm{HCC}=$ hepatocellular carcinoma. 
contrast agent Gd-EOB-DTPA compared with MDCT contrast agents.

In this study, the lesions with a diameter $>2 \mathrm{~cm}$ were detected using Gd-EOB-DTPA DCE-MRI and MDCT, while some lesions $<2 \mathrm{~cm}$ cannot be detected by MDCT. All lesions with a diameter not $>2 \mathrm{~cm}$ detected by MDCT can also be detected by Gd-EOBDTPA DCE-MRI. Some lesions $<2 \mathrm{~cm}$ detected by Gd-EOBDTPA DCE-MRI cannot be diagnosed by MDCT. Only using MDCT may therefore result in missed diagnosis and sub-optimal surgery time for HCC patients. Moreover, the missed diagnosis of smaller lesions in liver deprives some patients of the optimal operation schemes which can significantly affect survival and prognosis. ${ }^{[1]}$ Gd-EOB-DTPA DCE-MRI is helpful in detecting additional small lesions in patients diagnosed with HCC by MDCT.

Finally, it is important to acknowledge the limitations of this study. First, lesions which were considered to be non-HCC before surgery were not included; second, as this is a retrospective study, post-operative follow-up was not included and so post-operative recurrence was unknown; third, only patients who underwent surgery and had pathological outcomes were included, which may introduce selection bias. In conclusion, further studies are required to explore further the requirement for MRI detection in patients definitively diagnosed with HCC by enhanced CT.

\section{Conclusions}

Gd-EOB- DTPA DCE-MRI performed prior to surgery on patients with HCC is helpful for finding small HCC lesions in liver.

\section{Author contributions}

Conceptualization: Jiangfa Li, Songqing He.

Data curation: Xiaoqing Li, Jun Weng.

Formal analysis: Jiangfa Li, Zhenghang Li.

Funding acquisition: Songqing He.

Investigation: Liping Lei, Jianhua Gong, Junyi Wang, Longmiao Zhang.

Methodology: Jiangfa Li, Jun Weng.

Writing - original draft: Jiangfa Li, Xiaoqing Li.

Writing - review \& editing: Songqing He.

\section{References}

[1] Kim H-D, Lim Y-S, Han S, et al. Evaluation of early-stage hepatocellular carcinoma by magnetic resonance imaging with gadoxetic acid detects additional lesions and increases overall survival. Gastroenterology 2015;148:1371-82.

[2] Huppertz A, Balzer T, Blakeborough A, et al. Improved detection of focal liver lesions at MR imaging: multicenter comparison of gadoxetic acidenhanced MR images with intraoperative findings. Radiology 2004;230:266-75.

[3] Kudo M. Will Gd-EOB-MRI change the diagnostic algorithm in hepatocellular carcinoma? Oncology 2010;78(Suppl 1):87-93.

[4] Golfieri R, Renzulli M, Lucidi V, et al. Contribution of the hepatobiliary phase of Gd-EOB-DTPA-enhanced MRI to dynamic MRI in the detection of hypovascular small $(<2 \mathrm{~cm}) \mathrm{HCC}$ in cirrhosis. Eur Radiol 2011;21:1233-42.

[5] Lowenthal D, Zeile M, Lim WY, et al. Detection and characterisation of focal liver lesions in colorectal carcinoma patients: comparison of diffusion-weighted and Gd-EOB-DTPA enhanced MR imaging. Eur Radiol 2011;21:832-40.

[6] Yoo SH, Choi JY, Jang JW, et al. Gd-EOB-DTPA-enhanced MRI is better than MDCT in decision making of curative treatment for hepatocellular carcinoma. Ann Surg Oncol 2013;20:2893-900.

[7] Bottcher J, Hansch A, Pfeil A, et al. Detection and classification of different liver lesions: comparison of Gd-EOB-DTPA-enhanced MRI versus multiphasic spiral CT in a clinical single centre investigation. Eur J Radiol 2013;82:1860-9.

[8] Baek CK, Choi JY, Kim KA, et al. Hepatocellular carcinoma in patients with chronic liver disease: a comparison of gadoxetic acid-enhanced MRI and multiphasic MDCT. Clin Radiol 2012;67:148-56.

[9] Ahn SS, Kim MJ, Lim JS, et al. Added value of gadoxetic acid-enhanced hepatobiliary phase MR imaging in the diagnosis of hepatocellular carcinoma. Radiology 2010;255:459-66.

[10] Di Martino M, Marin D, Guerrisi A, et al. Intraindividual comparison of gadoxetate disodium-enhanced MR imaging and 64-section multidetector CT in the detection of hepatocellular carcinoma in patients with cirrhosis. Radiology 2010;256:806-16.

[11] Akai H, Kiryu S, Matsuda I, et al. Detection of hepatocellular carcinoma by Gd-EOB-DTPA-enhanced liver MRI: comparison with triple phase 64 detector row helical CT. Eur J Radiol 2011;80:310-5.

[12] Pascolo L, Cupelli F, Anelli PL, et al. Molecular mechanisms for the hepatic uptake of magnetic resonance imaging contrast agents. Biochem Biophys Res Commun 1999;257:746-52.

[13] Kim YK, Kim CS, Han YM, et al. Comparison of gadoxetic acidenhanced MRI and superparamagnetic iron oxide-enhanced MRI for the detection of hepatocellular carcinoma. Clin Radiol 2010;65:358-65.

[14] Kim YK, Kim CS, Han YM, et al. Detection of small hepatocellular carcinoma: can gadoxetic acid-enhanced magnetic resonance imaging replace combining gadopentetate dimeglumine-enhanced and superparamagnetic iron oxide-enhanced magnetic resonance imaging? Invest Radiol 2010;45:740-6.

[15] Inoue T, Kudo M, Komuta M, et al. Assessment of Gd-EOB-DTPAenhanced MRI for HCC and dysplastic nodules and comparison of detection sensitivity versus MDCT. J Gastroenterol 2012;47:1036-47.

[16] Lee JM, Zech CJ, Bolondi L, et al. Consensus report of the 4th international forum for gadolinium-ethoxybenzyl-diethylenetriamine pentaacetic acid magnetic resonance imaging. Korean J Radiol 2011;12:403-15.

[17] Kudo M, Matsui O, Sakamoto M, et al. Role of gadoliniumethoxybenzyl-diethylenetriamine pentaacetic acid-enhanced magnetic resonance imaging in the management of hepatocellular carcinoma: consensus at the symposium of the 48th Annual Meeting of the Liver Cancer Study Group of Japan. Oncology 2013;84(Suppl 1):21-7.

[18] Bruix J, Sherman M. Management of hepatocellular carcinoma: an update. Hepatology 2011;53:1020-2.

[19] Maiwald B, Lobsien D, Kahn T, et al. Is 3-Tesla Gd-EOB-DTPAenhanced MRI with diffusion-weighted imaging superior to 64-slice contrast-enhanced CT for the diagnosis of hepatocellular carcinoma? PLoS One 2014;9:e111935.

[20] Marin D, Di Martino M, Guerrisi A, et al. Hepatocellular carcinoma in patients with cirrhosis: qualitative comparison of gadobenate dimeglumine-enhanced MR imaging and multiphasic 64-section CT. Radiology 2009;251:85-95.

[21] Hwang J, Kim SH, Lee MW, et al. Small $(\leq 2 \mathrm{~cm})$ hepatocellular carcinoma in patients with chronic liver disease: comparison of gadoxetic acid-enhanced 3.0 T MRI and multiphasic 64-multirow detector CT. Br J Radiol 2012;85:e314-22.

[22] Kudo M. The 2008 Okuda lecture: Management of hepatocellular carcinoma: from surveillance to molecular targeted therapy. J Gastroenterol Hepatol 2010;25:439-52. 